\title{
An Aggregation Method for Mission Reliability of Equipment Support Object System
}

\author{
FENG Huijuan $^{1, a}$, LIU Xiangkai ${ }^{1, b}$, YANG Wancheng ${ }^{1, c}$ \\ ${ }^{1}$ Department of Automobile Engineering, Military Transportation University, Tianjin 300161, China \\ abluebellfhj@163.com, ${ }^{b}$ liu_xkai@126.com, ${ }^{\mathrm{t} t j}$ _ywc@sina.com
}

Keywords: multi-resolution modeling; aggregation; mission reliability; equipment support

\begin{abstract}
Mission reliability is an important parameter of equipment support object system. This paper proposed an aggregation method for mission reliability of equipment support object system: Firstly this paper studied the mission reliability aggregation method of single equipment and multi-equipment, and on this basis studied the mission reliability aggregation method of operational unit. At last, an example was given to show practicability of the method.
\end{abstract}

\section{Introduction}

Equipment Support System is an organic system which is formed from the interrelation and interaction of every level of support commands and items so as to accomplish the supporting task for combat units under a stated support environment ${ }^{[1]}$. Generally, it includes the resources of equipments, personals, parts and ammunitions, and the actions of maintenance, supply, storage, training and readiness ${ }^{[2]}$. In order to analyze and research the theory of equipment support, we usually set up the model of equipment support system. But there are many complex characteristics of this system, various methods from each aspect and level may be used to describe it. If we describe it from only one level, the model must be too simple, so a model with various levels and resolution, which can reflect the actions of it from different levels, need to be set up.

It is an important work to establish of a support object model with different resolution in equipment support multi-resolution modeling. Equipment support object system is also a complicated organic system consisting of various combat units. Combat unit is a military item of an organized structure which can accomplish combat and training tasks, such as a missile battalion or an anti-air artillery battalion. Combat unit generally consisted of a group of weapon systems and corresponding maintenance system. There are two kinds of methods to build a multi-resolution model of equipment support object system, one is support object entity (combat unit) oriented, and another is support object system parameters oriented. Parameters oriented modeling is a method based on the hierarchical breakdown and aggregation of the parameters. Task reliability is a basic parameter of an equipment support object system. The mission reliability of a combat unit is the capacity to continues perform mission under its own support conditions and during a stated times. It is a parameter of combat unit to reflect the capacity of continues performing mission. It is also an important parameter to reflect the capacity of maintenance support of a combat unit. The aggregation of task reliability is belonging to parameter oriented.

In this paper, the aggregation method for mission reliability of equipment support object system is from the part level. First, the mission reliability aggregation method of single equipment and multi-equipment has been studied, then a mission reliability aggregation method of combat unit has been presented, at last, an example was given to show the practicability of the method.

\section{Problem description}

There are many factors to influence the task reliability of equipment object system, such as the quality characteristics of weapons and equipments, and all factors of maintenance support system(e.g. maintenance persons, maintenance devices and spare parts), and the missions of the system itself. 
In order to simplify the model, in this paper, some hypothesis must be set up. Firstly, the repairer can repair all failure equipment by replacement during the task. Secondly, when a component is failure, if there is a spare part, it will be replaced immediately, and the replace time will be ignored. Finally, the replaced failure part can't be repaired in the operation site, all failure event is independent each other, and the repairer are plenty of in amount.

\section{Aggregation method}

\section{Definition of task reliability}

The mission reliability of a combat unit, is the probability of it to continues perform a stated mission under the maintenance support conditions during a stated times. Suppose the times of continues performing mission of a combat unit is $\mathrm{X}$, which is a random variable. Its task reliability is

$$
R(t)=P\{X \geq t\}
$$

\section{Mission reliability of a single equipment}

a. A single kind of component of an equipment. Supposing there are $N$ components of a same type in an equipment, which only $k$ need to be functioning for system success, denoted by $k / N(G)$. This equipment is a series system while $k=N$, and a parallel system while $k=1^{[3]}$. The failure times of every component obeys an exponential distribution with the parameters $\lambda$, and the amount of spare parts during mission is $s$.

The amount of failure components at $(t \geq 0)$ is expressed as $N(t)$, which is the status variable of system.

Let $n=s+N-k+1$, then:

The status space of system is $s=\{0,1, n, n\}$; The normal status set is $W=\{0,1=n-1\}$; And the failure status set is $F=\{z+n-k+1\}$.

The system status transition figure is shown in Fig. $1^{[4]}$.

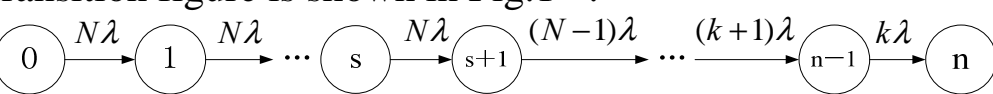

Fig. 1 system status transition figure (single equipment)

The transition ratio of system from status $i-1(i=1,2, \ldots, n)$ to status $i$ is:

$$
\beta_{i}= \begin{cases}N A & 1 \leq t \leq z-1 \\ g+N-t+2 \alpha & z+23 t \leq n\end{cases}
$$

Let $T_{i}$ is the transition times from $i-1$ to $i$. Given $\tau_{\mathrm{n}}=\sum_{n}^{n} \tau_{i}^{n}$, Thus, $\tau_{8}=0$.

As every $T_{i}$ is independent with each other, and $T_{i}$ obeys exponent distribution with parameter $\beta_{i}$. So $\left\{0, t \geq 0\right.$ is a renewing process ${ }^{[5]}$. And the continues performing mission time $X$ is just equal to the time from status 0 to status $n$, i.e. $X=\tau_{r}$. The mission reliability of single equipment is

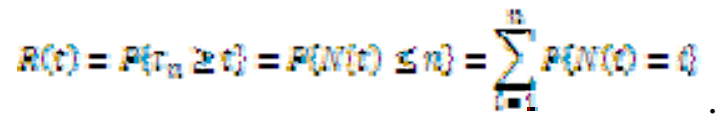

Given $P(t)=P\{(t)=t\}$, then

$$
R(t)=\sum_{i=1} P(t)
$$

According to the definition of renewing process ${ }^{[5]}$ and total probability formula, we can gained: $P_{i}(t+h)=P_{i}(t)\left(1-\beta_{i} h+o(h)\right)+P_{i-1}(t)\left(\beta_{i-1} h+o(h)\right)+o(h)=\left(1-\beta_{i} h\right) P_{i}(t)+\beta_{i-1} h P_{i-1}(t)+o(h)$

I.e.

$$
P_{i}(t+h)-P_{i}(t)=-\beta_{i} h P_{i}(t)+\beta_{i-1} h P_{i-1}(t)+o(h)
$$

From Eq.(4), and set $h \rightarrow 0$, then

$$
\begin{aligned}
& P_{1}^{\prime}(t)=-\beta_{1} P_{1}(t) \\
& P_{i}^{\prime}(t)=-\beta_{i} P_{i}(t)+\beta_{i-1} P_{i-1}(t)
\end{aligned}
$$

As $P_{1}(0)=1$, from Eq. (5) the following can be calculated $P_{1}(t)=\exp \left\{-\beta_{1} t\right\}$ 
Times both side of Eq. (6) with $\exp \left\{-\lambda_{i} t\right\}$, and through integral

$$
P_{i}(t)=\beta_{i-1} \exp \left\{-\beta_{i} t\right\} \int_{0}^{t} \exp \left\{\beta_{i} x\right\} P_{i-1}(x) \mathrm{d} x, 2 \leq i \leq n
$$

b. Multi kinds of components of a single equipment

In fact, while an equipment performing the mission, many kinds of components of it will be involved. As the relationship of various components are series in reliability, then

$R_{i}(t)=\prod_{i a i} R_{i}(t)$

While:

$R_{e}(t)$ : Mission reliability of the equipment;

$R_{i}$ : : Mission reliability of the equipment for $i t h$ components;

$I$ : Is the set of all kinds of components of the equipment.

\section{Mission reliability of multi same equipments}

Supposing there are an amount of $M$ equipments of the same type in a combat unit, mission requirement is that there are at least $r$ equipments being in operational conditions, otherwise, the mission will be failed.

In order to insure the most equipment are in operational status, when there are not enough spare parts to use, the parts can be disassembled from an disabled equipment, or an operational equipment(when the operational amount of this parts are more than minimum requirement) to replace the same kind of parts of another equipment.

The amount of failure components at $t(0)$ is expressed as $N(t)$, which is the status variable of system.

Let $p=s+M(N-k), q=s+M N-r k+1$, then The status space of system is:

$s=\{0,1, z, z+1, \ldots, z+1, \ldots q\}$

The normal status set is: $W=\{0,1, \ldots, s+1, z, \gamma+1, \ldots, q-1\}$

And the failure status set is: $F=\{n\}$

The system status transition figure is described in fig.2.

$$
\begin{aligned}
& \text { (0) } \stackrel{M N \lambda}{\longrightarrow} \stackrel{M N \lambda}{\longrightarrow} \cdots(\mathrm{s} \stackrel{M N \lambda}{\longrightarrow} \mathrm{s}+1 \stackrel{(M N-1) \lambda}{\longrightarrow} \cdots \stackrel{(M k+1) \lambda}{\longrightarrow} \mathrm{p} \stackrel{M k \lambda}{\longrightarrow} \mathrm{p}+1 \stackrel{(M-1) k \lambda}{\longrightarrow}
\end{aligned}
$$

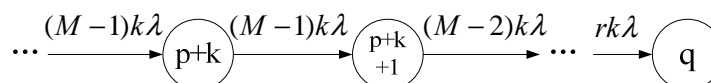

Fig. 2 system status transition figure(multi same equipments)

The transition ratio of system from status $i-1(i=1,2, \ldots, n)$ to status $i$ is:

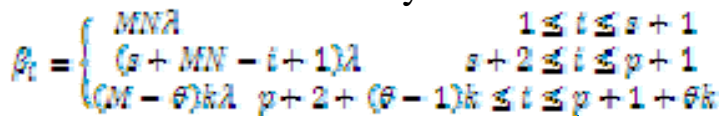

While:

$\theta$ is the amount of failure equipments, and $1 \leq \theta \leq M-r$

Let $T_{i}$ is the transition times from $i-1$ to $i$. Given $\tau_{q}=\sum_{a}^{q} \tau_{l}$, Thus, $\tau_{Q}=0$.

As every $T_{i}$ is independent with each other, and $T_{i}$ obeys exponent distribution with parameter $\beta_{i}$. So $w 0, t \geq 0\}$ is a renewing process. And the continues performing mission time $X$ is just equal to the time from status 0 to status $q$, i.e. $X=\tau_{\tau}$. The mission reliability of multi same equipment is:

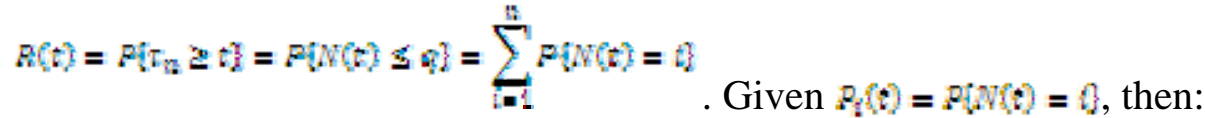

$$
\begin{aligned}
& R(t)=Q_{1} F_{t}(t)
\end{aligned}
$$

We can also calculate $F(t)$ through Eq. (5) and (7), and the system mission reliability of multi same equipment can be gained.

\section{Support system as basic operation unit}


Generally, a basic operational unit contains kinds of equipments, these equipments are complementary in functions, so series system model can be applied for them. The mission reliability of the basic operational unit is:

$R_{g}(t)=\prod_{l a z} F_{\varepsilon}(t)$

While:

$R_{\varepsilon}(t):$ Mission reliability of eth equipment;

$R_{g}$ tit: Mission reliability of the operational unit;

$E$ : Is the set of all kinds of equipment of the unit.

\section{Support object as operational unit}

Similarly, operational unit contains kinds of basic operational unit. There are stated mission reliability relationship between them, such as serial, parallel, standby redundant, parallel redundant, K-Out-Of-N, etc.,. The mission reliability of it is:

$R(t)=f\left(R_{g}(t)\right)$

While:

R(t): Mission reliability of the operational unit;

$\tilde{r}_{3}(\mathrm{t}$ : Mission reliability of the basic operational unit;

$f$ : Is a function of the mission reliability of every basic operational unit.

\section{Application and calculations}

A anti-air artillery unit is to be applied for an operational unit. It consists of a missile battalion and a anti-air artillery company. These two basic combat unit can exploit their own advantages, and make up each other. From the view of operational unit, these two operational units are formed to a serial system. The missile battalion contains equipment $\mathrm{A}(1)$, equipment $\mathrm{B}(1)$, and equipment $\mathrm{C}(3)$. The anti-air artillery company contains equipment $\mathrm{D}(1)$ and equipment $\mathrm{E}(6)$. According to the requirement of the mission, equipment $\mathrm{A}, \mathrm{B}$ and $\mathrm{D}$ must be operational. There are at least 2 equipments of $\mathrm{C}$, and 4 equipments of $\mathrm{E}$. The basic construction and requirement of the operational unit, and the critical parts of them have been described in Fig. 3, Fig.4, and Table 1.

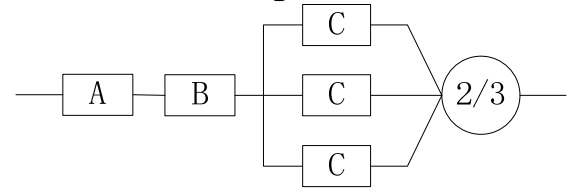

Fig.3 Mission reliability diagram of missile battalion

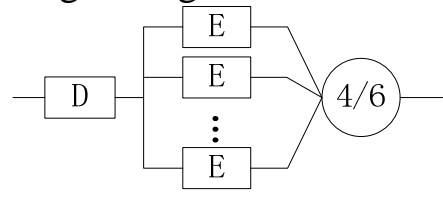

Fig.4 Mission reliability diagram of anti-air artillery company

Table 1 structure, failure, and requirement data of the equipments

\begin{tabular}{|c|c|c|c|c|c|}
\hline equipment & $r / M$ & Part & $k / N$ & $s$ & $\lambda$ \\
\hline \multirow{2}{*}{$\mathrm{A}$} & \multirow{2}{*}{$1 / 1$} & $\mathrm{~A} 1$ & $1 / 2$ & 3 & 0.008 \\
\cline { 3 - 6 } & & $\mathrm{A} 2$ & $1 / 3$ & 2 & 0.004 \\
\hline $\mathrm{B}$ & $1 / 1$ & $\mathrm{~B} 1$ & $2 / 3$ & 3 & 0.005 \\
\hline \multirow{2}{*}{$\mathrm{C}$} & \multirow{2}{*}{$2 / 3$} & $\mathrm{C} 1$ & $1 / 1$ & 6 & 0.003 \\
\cline { 3 - 6 } & & $\mathrm{C} 2$ & $2 / 3$ & 6 & 0.010 \\
\hline $\mathrm{D}$ & $1 / 1$ & $\mathrm{D} 1$ & $1 / 2$ & 2 & 0.003 \\
\hline $\mathrm{E}$ & $4 / 6$ & $\mathrm{E} 1$ & $1 / 2$ & 6 & 0.005 \\
\hline
\end{tabular}

By applying the aggregation method, using the software of Mathamatica, the mission reliability functions have been calculated, and the mission reliability curves have also been drawn, shown as the fig.4 and fig.7. 


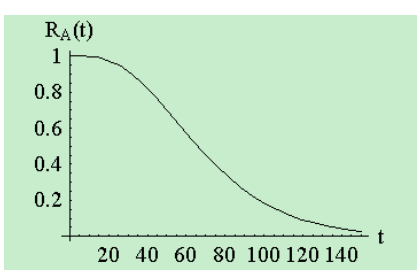

Fig.4 Mission reliability curve of equipment A

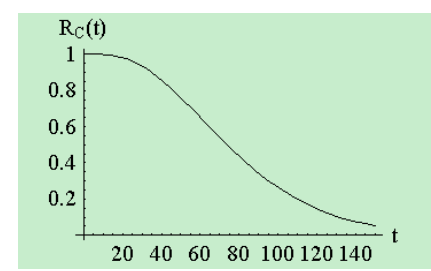

Fig.6 Mission reliability curve of equipment C(3)

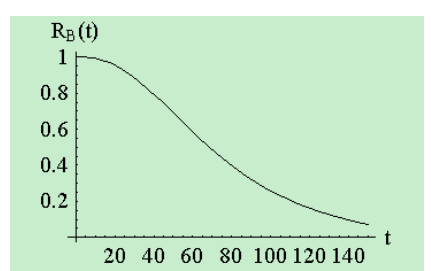

Fig.5 Mission reliability curve of equipment B

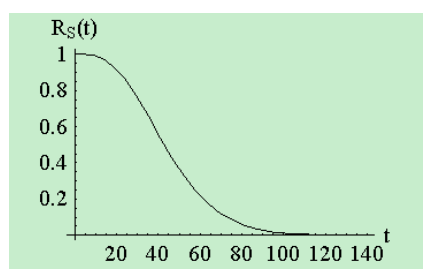

Fig.7 Mission reliability curve of the operational unit

\section{Conclusions}

From the modeling of equipment support of multi-resolution, regarding the weapon system and maintenance support system as an organic system, the aggregation method of multi-resolution for mission reliability of the operational unit during the training and combat task have been researched. The aggregation methods of Mission reliability for a single equipment, multi same equipment, and operational unit have been presented, and their reliability mathematic models have been established. An anti-air combat unit has been used to apply and verify the methods. The results indicated that, the method can set up a basis on resolving the problem of system mission reliability modeling, and also lay a foundation for the multi-resolution of equipment support system.

\section{References}

[1] LUO Cheng. Research on Multi-level Simulation and Modeling of Equipment Support. Doctoral Dissertation, Ordnance Engineering College, 2006

[2] WANG Dongnan. Modeling and Simulaiton of Task-Oriented Evaluation of Maintenance Support Caability. Doctoral Dissertation, National University of Defense Technology, 2005

[3] GAN Maozhi. Military Equipment Maintenance Engineering. National Defense Industry Press, 1999

[4] CAO Jinhua. Reliability Mathematics Introduction. Science Press, 1986

[5] DENG Yonglu. Random Point Process and Application, Science Press, 1992 